\title{
Intoxicação por organofosforados em búfalos (Bubalus bubalis) no Rio Grande do Sul ${ }^{1}$
}

\author{
Fabiane Borelli Grecco², Ana Lucia Schild ${ }^{3 *}$, Mauro Pereira Soares ${ }^{3}$, \\ Margarida Buss Raffi ${ }^{4}$, Eliza Simone Viégas Sallis ${ }^{4}$ e Maria Cecília Damé ${ }^{5}$
}

\begin{abstract}
Grecco F.B., Schild A.L., Soares M.P., Raffi M.B., Sallis E.S.V. \& Damé M.C. 2009. [Organophosphate poisoning in buffaloes (Bubalus bubalis) in southern Brazil.] Intoxicação por organofosforados em búfalos (Bubalus bubalis) no Rio Grande do Sul. Pesquisa Veterinária Brasileira 29(3):211-214. Laboratório Regional de Diagnóstico, Faculdade de Veterinária, Universidade Federal de Pelotas, Campus Universitário s/n, Pelotas, RS 96010-900, Brazil. E-mail: alschild@terra.com.br

This paper describes organophosphate (ORF) poisoning in a herd of water buffaloes (Bubalus bubalis) in southern Brazil, which were treated with a single dose of $12 \mathrm{mg} / \mathrm{kg}$ body weight of Expertan ${ }^{\circledR}$ pour-on (chlorpyriphos) to control ectoparasites. Clinical signs, observed 7-45 days after exposure, were diarrhea, hypersalivation, ataxia, muscular tremors, weakness of pelvic limbs, paresis and flaccid paralysis and lateral recumbence. Out of 267 buffaloes 61 died. Necropsy of three animals that died 24-72 hours after onset of clinical signs, revealed congestion and serosal hemorrhages scattered along the bowel, emphysema and edema of the lungs. No significant histopathological changes were found. Residues of chlorpyriphos were detected in liver, kidneys and nervous system of the one necropsied buffalo. Despite the absence of histological lesions in the central and peripheral nervous system, the epidemiological, clinical, gross and toxicological findings suggest delayed neurotoxicity induced by organophosphates.
\end{abstract}

INDEX TERMS: Diseases of water buffaloes, poisoning, organophosphate toxicosis.

RESUMO.- Descreve-se um surto de intoxicação por organofosforados (ORFs) em búfalos (Bubalus bubalis) na região sul do Rio Grande do Sul. Os animais foram submetidos à aplicação de Expertan® pour-on (clorpirifós) na dose de $12 \mathrm{mg} / \mathrm{kg}$ de peso animal para controle de infestação por piolhos e desenvolveram sinais clínicos de intoxicação em um período variável entre 7-45 dias após a exposição ao produto. Os sinais clínicos caracterizaram-se por anorexia, diarréia, salivação intensa, incoor-

\footnotetext{
${ }^{1}$ Recebido em 7 de outubro de 2008.

Aceito para publicação em 23 de outubro de 2008.

2 Pós-Graduanda em Medicina Veterinária, Faculdade de Veterinária, Universidade Federal de Pelotas (UFPel).

${ }^{3}$ Laboratório Regional de Diagnóstico, Faculdade de Veterinária, UFPel, Campus Universitário s/n, Pelotas, RS 96010-900, Brasil. *Autor para correspondência: alschild@ terra.com.br

${ }^{4}$ Departamento de Patologia, Faculdade de Veterinária, UFPel, Pelotas, RS.

5 Embrapa Clima Temperado, Rodovia BR 392, km 78, Cx. Postal 403, Capão do Leão, Pelotas, RS 96001-970.
}

denação motora, tremores musculares, paresia e paralisia flácida, decúbito lateral e morte. Em conseqüência da intoxicação morreram 61 búfalos de um total de 267 animais sob risco. Foram realizadas três necropsias de búfalos que morreram 24-72 horas após o início dos sinais clínicos. As lesões macroscópicas caracterizaram-se por congestão e hemorragias intestinais, enfisema e edema pulmonares. Não foram observadas alterações microscópicas significativas em nenhum dos animais. Apesar da ausência de alterações histológicas no sistema nervoso central e periférico, os achados epidemiológicos, clínicos, macroscópicos e toxicológicos sugerem o diagnóstico de neurotoxicidade tardia induzida por ORFs.

TERMOS DE INDEXAÇÃO: Doenças de búfalos, intoxicações, toxicose por organofosforados.

\section{INTRODUÇÃO}

Compostos organofosforados (ORFs) e carbamatos fazem parte de um grupo de substâncias utilizadas amplamente como inseticidas, na agricultura, como anti-helmín- 
ticos ou no controle de ectoparasitas em algumas espécies domésticas. Os ORFs mais frequentemente utilizados são metaminofós, dimixion, fenitrotion, fention, fosfomet, triclorfon, clorfenrifós, ethion, diazinon e clorpirifós. Com menor freqüência são utilizados os carbamatos carbaril, aldicarb, carbofuran, methomyl, popoxur, oxamil, pirimidicarb e dioxicarb (Osweiler 1998, Barbosa et al. 2005). Esses produtos são rapidamente absorvidos por ingestão, inalação, absorção percutânea e pela conjuntiva (Radostitis et al. 2000) sendo que a taxa de absorção cutânea é influenciada pelo solvente utilizado (Barros et al. 2006).

O uso inadequado dos ORFs pode causar grandes prejuízos na pecuária e a intoxicação ocorre por contaminação da água, alimentos, pastagens, preparação e uso de concentrações excessivas do produto (Boermans et al.1984, Radostits et al. 2000). Alguns fatores de risco para a ocorrência da intoxicação têm sido mencionados como idade (animais jovens são sensíveis), estresse, privação de água, frio intenso e sexo (fêmeas prenhes e machos inteiros maiores de 8 meses). São também fatores de risco os métodos de aplicação, p.e. aplicação pour on tem efeito retardado em até 24 horas se comparada com a pulverização (Radostitis et al. 2000). Alguns produtos são mais tóxicos para as fêmeas como o diclorvos, dioxation e paration e outros possuem toxicidade maior para machos como o clorpirifós, dimetoato e fention e não devem ser utilizados em animais maiores de 8 meses devido a interação com a testosterona (Radostitis et al. 2000).

A dose tóxica dos ORFs varia de acordo com o produto (Barros et al. 2006). Em trabalhos de intoxicação experimental tem sido demonstrado que a ordem de toxicidade desses produtos difere amplamente entre búfalos e outras espécies domésticas e que a extrapolação de dados sobre doses utilizadas em bovinos não deve ser feita para bubalinos (Raina et al. 1990). Diclorvós na dose de $160 \mathrm{mg}$ via oral para búfalos jovens causou sinais clínicos em 4-10 minutos após administração (Raina et al. 1990).

No Brasil há descrição de surtos da intoxicação por ORFs em bovinos em Santa Catarina (Gava 2001), no Pará (Barbosa et al. 2003) e no Distrito Federal (Castro et al. 2007) e por carbamatos no Rio Grande do Sul (Soares et al. 2002). Entre os bovinos parece haver uma suscetibilidade maior das raças zebuínas à intoxicação (Barros \& Driemeier 2007). Intoxicação por ORFs em búfalos foi mencionada por Damé (2000) em um lote de 7 animais machos dos quais um morreu 5 dias após o início dos sinais clínicos de apatia e salivação que iniciaram uma semana após tratamento com triclorfon.

Muitos ORFs inibem a atividade da enzima acetilcolinesterase. O efeito tóxico agudo destes compostos se origina dessa inibição pela formação de complexos com a acetilcolinesterase levando ao acúmulo de acetilcolina nas sinapses, junções neuromusculares e outros sítios neuroefetores (Johnson 1975, Thompson et al. 1993). As primeiras alterações clínicas surgem em 1-2 horas após a exposição ao produto e nos casos não-letais a recuperação ocorre em até 48 horas. A intoxicação crônica ocorre quando o produto é aplicado de forma repetida em intervalos de alguns dias, observando-se sinais clínicos moderados (Gava 2001).

Alguns ORFs, como clorpirifós, diclorvós, paration e triclorfon os produzem um efeito tóxico não-relacionado à capacidade de inibir a acetilcolinesterase, denominado neurotoxicidade tardia que se caracteriza por ataxia e paralisia dos membros (Johnson 1975). Ocorre axonopatia distal com morte de neurônios, especialmente dos grandes neurônios da medula espinhal e seus processos (McConnell et al. 1999). Essa forma de intoxicação tem sido relacionada a uma enzima-alvo, denominada esterase neurotóxica, resultando na alteração da velocidade de transmissão da excitação ao longo dos nervos periféricos. Paresia e paralisia são observadas no mínimo 8 dias após a exposição ao produto (Johnson 1975, Radostits et al. 2000) e neste período a atividade da enzima alvo pode ser normal (Thompson et al. 1993). Esta forma de intoxicação foi reproduzida experimentalmente em ovinos que adoeceram 16-25 dias após a administração de haloxon via oral (Souza et al. 1996).

O acúmulo de acetilcolina induz três tipos de sinais clínicos: (1) muscarínicos, caracterizados por sudorese, sialorréia, hipermotilidade gastrintestinal, diarréia, tenesmo e bradicardia; (2) nicotínicos, resultantes da ação da acetilcolina sobre as placas motoras e caracterizados por rigidez muscular, tremores paresia e paralisia e (3) do sistema nervoso central que resultam em inquietação, ataxia, convulsão, depressão e coma (Barros \& Driemeier 2007). Os sinais clínicos de ação tardia são caracterizados por dificuldade locomotora que progride para paresia e paralisia. A morte ocorre por insuficiência cardíaca e respiratória.

$\mathrm{Na}$ forma aguda há pouca diferença entre os sinais clínicos produzidos por diferentes compostos, mas a via de absorção pode estimular a ocorrência de um ou outro tipo de sinal (Barros \& Driemeier 2007).

Não há lesões macroscópicas características embora congestão e hemorragias intestinais, congestão e edema pulmonar e hemorragias na bexiga (Barbosa et al. 2005) possam ser observados. Microscopicamente, na intoxicação crônica podem ser observados edema axonal, esferóides axonais, cromatólise e necrose neuronal principalmente no tronco encefálico e medula espinhal (Summers et al. 1995, Gava 2001). Os objetivos deste trabalho foram descrever um surto intoxicação por ORFs em bubalinos na região sul do Rio Grande do Sul.

\section{MATERIAL E MÉTODOS}

Os dados epidemiológicos e os sinais clínicos foram obtidos em visita às propriedades ou fornecidos pelo médico veterinário responsável pelo rebanho. Três búfalos afetados que morreram entre 8 e 10 dias após a aplicação do medicamento, foram necropsiados. Fragmentos de todos os órgãos foram fixados em formalina $10 \%$, processados rotineiramente e corados 
pela hematoxilina e eosina ( $\mathrm{HE})$. Os seguintes cortes transversais do sistema nervoso central foram processados rotineiramente para histopatologia: córtex frontal, núcleos da base, tálamo, córtex parietal, colículos rostrais, córtex occipital, ponte, pedúnculos cerebelares, cerebelo, bulbo na altura do óbex, medula cervical. Do sistema nervoso periférico foram colhidos para histopatologia nervos paravertebrais e cranianos. Fragmentos de fígado, rins, sistema nervoso central, conteúdo ruminal e intestinal de um dos animais necropsiados foram enviados ao Laboratório de Análises Toxicológicas Ltda (Latox), Porto Alegre, RS, para análise da presença de ORFs pelas técnicas de cromatografia em camada delgada e cromatografia gasosa 6 .

Após o diagnóstico da intoxicação os animais foram tratados com sulfato de atropina na dose de $15 \mathrm{ml}$ para cada animal uma a duas vezes por dia. Os búfalos receberam, ainda, tratamento de suporte com antitóxicos, fluidoterapia e antibiótico Riverin®.

\section{RESULTADOS}

\section{Epidemiologia}

O surto ocorreu em junho de 2008 em um lote de 267 búfalos (261 fêmeas e 6 machos) de uma propriedade localizada no município de Pelotas, RS. Nesses búfalos foi aplicado pour on de clorpirifós [0,0-dietil 0-(3,5,6tricloro-2-piridil)-fósforo-tionato] na dose de $10 \mathrm{ml} / 100 \mathrm{~kg}$ de peso animal $(12 \mathrm{mg} / \mathrm{kg})$ para combate a infestação por piolhos. O peso dos animais foi calculado por estimativa entre $500-600 \mathrm{~kg}$. Os primeiros animais afetados apresentaram os sinais clínicos sete dias após a aplicação do medicamento e os últimos 45 dias após. A evolução da enfermidade variou de 24 horas a 5 dias, ocorrendo a morte de 61 animais, cinco machos de 2,5 anos e 56 fêmeas com idades entre 2,5-20 anos, das quais aproximadamente $60 \%$ estavam prenhes.

\section{Sinais clínicos}

Os búfalos intoxicados apresentavam inicialmente anorexia, salivação excessiva, diarréia e andar trôpego. Com a evolução da enfermidade havia desidratação, agressividade, tremores musculares, paresia e paralisia flácida, principalmente dos membros pélvicos, decúbito lateral e morte. Não foi possível estabelecer quantos búfalos que adoeceram se recuperaram após o tratamento com sulfato de atropina.

\section{Patologia}

Foram necropsiados três búfalos e as lesões macroscópicas caracterizaram-se por congestão e hemorragias intestinais, enfisema e edema pulmonar. Não foram observadas lesões histológicas no sistema nervoso central e nervos periféricos estudados.

Nas amostras dos tecidos enviados ao Latox foram encontrados resíduos de ORFs no sistema nervoso central na concentração de $0,20 \mathrm{ig} / \mathrm{kg}$ no sistema nervoso central e $0,30 \mathrm{ig} / \mathrm{kg}$ em um pool de fígado e rins.

\footnotetext{
${ }^{6}$ Adriana Wolffenbütten, Química toxicológica, Laboratório de Toxicologia (Latox), Porto Alegre, RS.
}

\section{DISCUSSÃO}

O diagnóstico da intoxicação por ORFs (clorpirifós) foi realizado com base no histórico da aplicação do produto pour on, sinais clínicos, lesões macroscópicas e pela presença de resíduos do produto no sistema nervoso central, fígado e rins de um dos animais mortos.

As manifestações clínicas observadas foram características da inibição da colinesterase com sinais muscarínicos, nicotínicos e do sistema nervoso central, sendo evidenciados 7-45 dias após à exposição ao produto. Aparentemente ocorreu a forma denominada de neurotoxicidade tardia induzida por ORFs. A neurotoxicidade tardia tem sido descrita em humanos e animais produzindo uma síndrome paralítica associada a axonopatia distal que se manifesta 1-4 semanas até poucos meses após a exposição dependendo do composto utilizado, dose, freqüência da exposição e do solvente (Summers et al. 1995). A neurotoxicidade tardia é produzida por alguns ORFs incluindo o clorpirofós e os sinais clínicos observados são de paresia e paralisia (Johnson 1975). Por outro lado, são descritos também, outros sinais como diarréia, anorexia, depressão, estase ruminal (Radostitis et al. 2000), que foram observados nos búfalos deste relato. Em humanos é descrita uma forma intermediária da intoxicação cujos sinais clínicos aparecem 1-5 dias após os efeitos agudos (crise colinérgica) e antes das manifestações de neurotoxicidade tardia (Peyon 2007).

A aplicação pour-on de medicamentos é utilizada para liberação lenta de pequenas quantidades, o que neste caso pode ter contribuído para o aparecimento dos sinais clínicos até 45 dias após a aplicação do medicamento, associada ao peso dos animais que foi apenas estimado o que poderia ter levado a dosagens incorretas do produto.

A dose administrada aos búfalos de $12 \mathrm{mg} / \mathrm{kg}$ de peso animal é a recomendada para bovinos tratados com clorpirifós, o que reforça que bubalinos, aparentemente são mais sensíveis a esse produto do que os bovinos. Tem sido mencionado que ORFs não devem ser utilizados em bubalinos na dose recomendada para bovinos já que a sensibilidade dessa espécie a esses produtos é variável e que tais doses não são seguras para a administração em bubalinos (Raina et al. 1990). Trabalhos experimentais nessa espécie demonstraram que $160 \mathrm{mg} /$ $\mathrm{kg}$ de peso animal de diclorvós administradas oralmente (Raina \& Malik 1991), e 100-125mg/kg de peso animal de malathion (Gupta, 1984) causaram sinais clínicos de intoxicação aguda em poucos minutos à uma hora após a exposição aos produtos, respectivamente.

Não foram observados sinais de intoxicação aguda nos búfalos deste trabalho. Segundo alguns autores a maioria dos ORFs é rapidamente metabolizada e as intoxicações subaguda ou crônica raramente são observadas, a não ser quando os animais recebem doses repetidas do produto (Thompson et al. 1993). Um surto de intoxicação crônica em bovinos foi relatado em Santa Catarina, após a administração repetida de clorfenifós e triclorfon com óleo queimado. Nessa oportunidade o curso clínico da 
enfermidade variou de 3 a 5 meses (Gava 2001). Nos búfalos do presente trabalho o curso clínico da doença variou de 24 horas a 5 dias, após uma única aplicação.

As lesões macroscópicas observadas nos búfalos deste trabalho caracterizadas principalmente por congestão e hemorragias no trato gastrintestinal, e enfisema e edema pulmonar, apesar de serem inespecíficas, têm sido descritas em outros surtos de intoxicação por ORFs independente da forma clínica observada (Boermans et al. 1984, Khan 2001, Barros et al. 2006). Histologicamente é mencionada a presença de edema axonal, cromatólise e necrose neuronal no tronco encefálico com esferóides axonais na substância branca nos casos de intoxicação crônica por esses produtos (Gava 2001). Na neurotoxicidade tardia induzida por ORFs são descritas alterações degenerativas nos nervos e degeneração neuroaxonal em núcleos do cerebelo, bulbo e trato espinhal do trigêmeo e medula espinhal (Summers et al. 1995, Souza et al. 1996) que não foram observados nos búfalos deste trabalho. Em casos agudos da intoxicação não tem sido observadas lesões histológicas significativas (Castro et al. 2007).

Apesar da ausência de lesões histológicas no sistema nervoso central e periférico dos búfalos deste trabalho o aparecimento dos sinais clínicos somente após 7-45 dias da exposição ao produto e à forma de aplicação do mesmo sugere a ocorrência da forma tardia da intoxicação. Em um relato de neurotoxicidade tardia em bovinos também não foram observadas lesões histológicas significativas no tecido nervoso e o diagnóstico foi feito com base na epidemiologia, sinais clínicos e demonstração de ORFs nos tecidos dos animais (Thompson et al. 1993).

Sulfato de atropina, utilizado após a confirmação do diagnóstico não foi eficiente para evitar as mortes dos búfalos tratados, não sendo possível determinar quantos animais tratados se recuperaram. Isso foi observado, também, na intoxicação por tribufós em bovinos e atribuído ao fato de que sulfato de atropina previne os efeitos muscarínicos, mas não os nicotínicos que causam paresia e paralisia (Pugh 1975, Boermans et al. 1984), os quais foram observados, também, nos búfalos deste relato.

A intoxicação por ORFs deve ser diferenciada de outras enfermidades que causam sinais clínicos neurológicos de paresia e paralisia dos membros posteriores e tremores musculares como intoxicação por Claviceps paspali que pode afetar vários animais em períodos curtos de tempo, raiva, botulismo e, também, infecção por Yersinia peseudotuberculosis, que causa diarréia.

A intoxicação por ORFs não é freqüente em nossa região; apesar disso, episódios podem ocorrer quando os animais são tratados com doses repetidas ou acima do recomendado. Uma vez que os búfalos são mais sensíveis à intoxicação por esses produtos do que os bovinos não é recomendada a utilização desses compostos nessa espécie animal.

\section{REFERÊNCIAS}

Barbosa J.D., Oliveira C.M., Duarte M., Cerqueira, V. \& Riet-Correa G.
2003. Intoxicação por organofosforados em rebanho de bovinos. $11^{\circ}$ Congresso Latino-Americano de Buiatria, Salvador, BA.

Barbosa J.D., Oliveira C.M.C., Duarte M.D. \& Albernaz T.T. 2005. Intoxicações com manifestações neurológicas em ruminantes. II Simpósio Mineiro de Buiatria, Belo Horizonte, MG.

Barros C.S.L., Driemeier D., Dutra I.S. \& Lemos A.A.R. 2006. Doenças do sistema nervoso de bovinos no Brasil. Coleção Vallée, São Paulo. 207p.

Barros C.S.L. \& Driemeier D. 2007. Intoxicação por organofosforados e carbamatos, p.80-85. In: Riet-Correa F., Schild A.L., Lemos R.A.A. \& Borge J.R.J. (Ed.), Doenças de Ruminantes e Eqüídeos. Vol.2. Pallotti Gráfica e Editora, Santa Maria, RS.

Boermans H.J., Black W.D., Chesney R. \& Robb S. 1984. Terbufos poisoning in a dairy herd. Can.Vet. J. (25):335-338.

Castro M.B.C., Moscardini A.R.C., Reis J.L., Novaes E.P.F. \& Borges J.R.J. 2007. Intoxicação aguda por diazinon em bovinos. Ciência Rural 37(5):1498-1501.

Damé M.C.F. 2000. Seleção, manejo e produtividade dos bubalinos da Embrapa Clima Temperado. Documentos, Embrapa Clima Temperado, Capão do Leão, RS. 31p.

Gava A. 2001. Intoxicação por organofosforados e carbamatos, p.3542. In: Riet-Correa F., Schild A.L., Lemos R.A.A. \& Méndez M.C. (Ed.), Doenças de Ruminantes e Eqüinos. $2^{\mathrm{a}}$ ed. Varela Editora e Livraria, São Paulo,

Gupta R. 1984. Acute malathion toxicosis na related enzymatic alterations in Bubalus bubalis: Antidotal treatment whit atropine, 2PAM, and diazepam. J. Toxicol. Environ. Hlth (14):291-303.

Johnson M.K. 1975. Organophosphorus esters causing delayed neurotoxic effects. Arch. Toxicology 34:259-258.

Khan O. 2001. Organophosphate poisoning in a group of replacement heifers and dry cows. Can. Vet. J. 42:561-563.

McConnell R., Téllez-Delgado E., Cuandra R., Tórres E., Keifer M., Almendárez J., Miranda J., Hassan A.N., Wolf M., Simpson D. \& Lundberg I. 1999. Organophosphate neuropathy due to methamidophos: Biochemical and neurophysiological markers. Arch. Toxicol. 73:296-300.

Osweiler G.D. 1998. Inseticidas e moluscicidas, p.259-282. In:__ (Ed.), Toxicologia Veterinária. Editora Artes Médicas Sul, Porto Alegre, RS.

Peyon F.C.A. 2007. Implementação de um bioindicador para a neuropatia induzida por organofosforados(OPDIN). Dissertação de Mestrado, Escola Nacional de Saúde Pública, Rio de Janeiro, RJ.

Pugh W.S. 1975. An outbreak of organophosphate poisoning (Thimet) in cattle. Can.Vet. J. 16:56-58.

Radostitis E.M., Gay C.C., Blood D.C. \& Hinchcliff K.W. 2000. Veterinary Medicine. 9th ed. W.B. Saunders, London. 1881p.

Raina R. \& Malik J.K. 1991. The therapeutic effects of 2,3-butanedione monoxime and atropine in severe dichlorvos intoxication in Buffalo calves. Vet. Res. Commun. 15(4):319-325.

Raina R., Srivastava K.A. \& Malik J.K.1990. Effects of repeated topical application of dicholorvos on blood enzymes and its toxicity in Buffalo calves (Bubalus bubalis). Brit. Vet. J. 146:264-269.

Soares A.C., Dias M.M., Traverso S.D. \& Driemeier D. 2002. Intoxicação acidental por carbamatos em bovinos de corte no sul do Brasil. 29ํㅡㄹ Congresso Brasileiro de Medicina Veterinária, Gramado, RS, p.714. (Resumo)

Souza M.V., Graça D.L. \& Cervo D. 1996. Neurotoxidade tardia experimentalmente induzida por haloxon em ovinos. Arq. Bras. Med. Vet. Zootec. 48(3): 275-286.

Summers B.A., Cummings J.F. \& de Lahunta A. 1995.Veterinary Neuropathology. Mosby-Year Books, St Louis, Missouri. 527p.

Thompson J.C., Thompson A.H. \& Thornton R.N. 1993. Accidental poisoning of a group of yearling cattle by the organophosphate insecticide trichloronat. N. Z. Vet. J. 41:87-90. 